\title{
Direct Atomic Force Microscopy Observation of DNA Tile Crystal Growth at the Single-Molecule Level
}

\author{
Constantine G. Evans, ${ }^{\dagger}$ Rizal F. Hariadi, ${ }^{\dagger, \perp}$ and Erik Winfree ${ }^{*} \S$
}

${ }^{\dagger}$ Physics, ${ }^{\ddagger}$ Applied Physics, and ${ }^{\S}$ Computer Science, Bioengineering, and Computation and Neural Systems, California Institute of Technology, Pasadena, California 91125, United States

\section{Supporting Information}
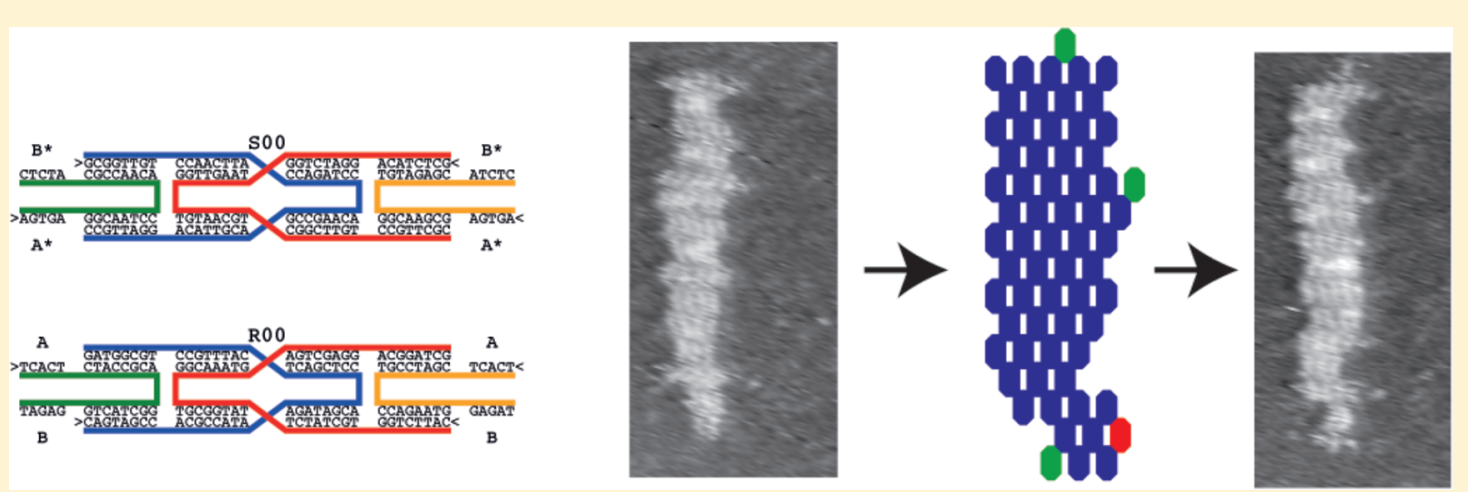

ABSTRACT: While the theoretical implications of models of DNA tile self-assembly have been extensively researched and such models have been used to design DNA tile systems for use in experiments, there has been little research testing the fundamental assumptions of those models. In this paper, we use direct observation of individual tile attachments and detachments of two DNA tile systems on a mica surface imaged with an atomic force microscope (AFM) to compile statistics of tile attachments and detachments. We show that these statistics fit the widely used kinetic Tile Assembly Model and demonstrate AFM movies as a viable technique for directly investigating DNA tile systems during growth rather than after assembly.

\section{INTRODUCTION}

Macromolecular self-assembly is common in nature as a mechanism for the construction of complex structures: the construction of microtubules from tubulin in the cytoskeleton is one notable example. Analogous self-assembling systems have been designed using a variety of often biology-derived "building blocks" to construct large, complex structures with nanoscale resolution suitable for a diverse range of applications. ${ }^{1-3}$

Crystals formed from DNA tiles are particularly interesting in their ability to self-assemble based on designed, single-stranded DNA "sticky ends" that attach to complementary ends on other tiles. ${ }^{4}$ This allows crystals to be designed that have complex, programmable structure despite consisting of only a relatively small number of different tile types. ${ }^{5-8}$

In this regard, the tiles are similar to Wang tiles, ${ }^{9}$ which were used as a basis for the abstract Tile Assembly Model (aTAM) of DNA tile assembly. ${ }^{10}$ In this model (see Figure 1), tiles fill empty lattice spaces if they can attach by at least a certain number of correct bonds, usually two. While the model ignores the kinetics of tile attachment and ignores detachment entirely, it does serve as a useful basis for designing the logic behind both simple and complex tile sets. In an attempt to make a model useful for understanding experimental results, the kinetic Tile Assembly Model (kTAM) was devised. ${ }^{10,11}$ In this model, free tiles in solution attach to empty binding sites at a rate determined only by their concentrations, and detach at a rate determined only by the number of matching sticky-end bindings holding them to the lattice-in other words, an onrate and off-rate per tile of

$$
\begin{aligned}
& k_{\text {on }}=k_{\mathrm{f}}[c] \\
& \text { and } \\
& k_{\text {off }}=k_{\mathrm{f}} e^{-b \Delta G_{\mathrm{se}}^{\circ} / R T+\alpha}
\end{aligned}
$$

where $k_{\mathrm{f}}$ is the forward rate constant, $[c]$ is the concentration of free tiles of the type of interest, $b$ is the number of correct bonds holding a particular tile in place, $\Delta G_{\mathrm{se}}^{\circ}$ is the standard free energy of a single bond, and $\alpha$ is a constant factor accounting for other binding energies. In order to remove $\alpha$, parameters $\hat{k}_{\mathrm{f}} \equiv k_{\mathrm{f}} e^{\alpha}$ and dimensionless "free energy" $G_{\mathrm{mc}}$ where $[c]=$ $e^{-G_{\mathrm{mc}}+\alpha}$ are used; these, along with a dimensionless energy $G_{\mathrm{se}} \equiv \Delta G_{\mathrm{se}}^{\circ} / R T$ result in

$$
\begin{aligned}
& k_{\text {on }}=\hat{k}_{\mathrm{f}} \mathrm{e}^{-G_{\mathrm{mc}}} \\
& \text { and } \\
& k_{\text {off }}=\hat{k}_{\mathrm{f}} \mathrm{e}^{-b G_{\mathrm{se}}}
\end{aligned}
$$

where both $G_{\mathrm{mc}}$ and $G_{\mathrm{se}}$ are usually positive numbers.

Received: February 13, 2012

Published: June 13, 2012 
aTAM

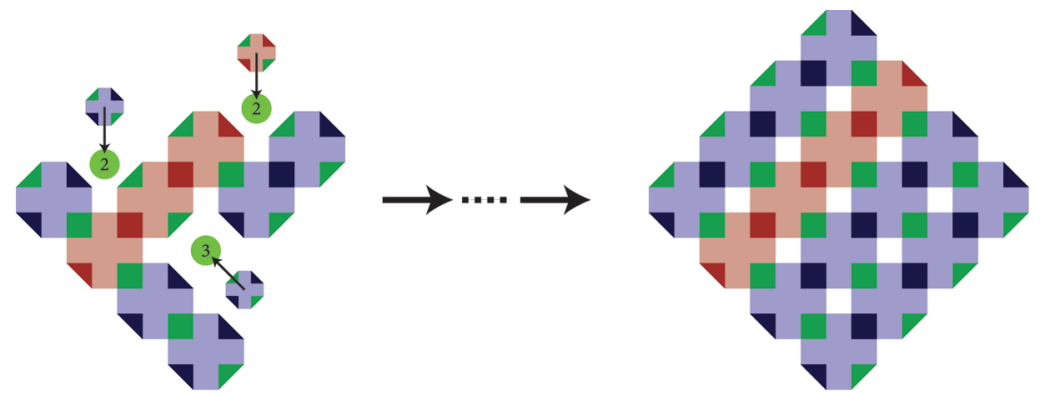

kTAM
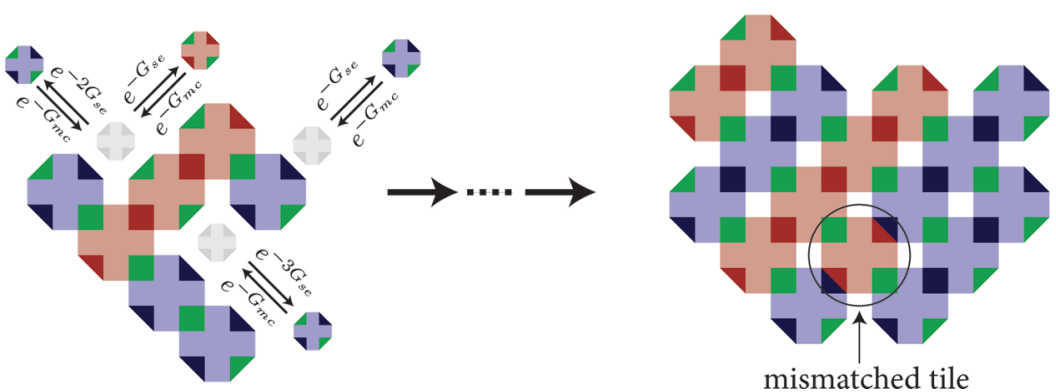

Figure 1. Illustrations of the abstract (aTAM) and kinetic (kTAM) Tile Assembly Models. In the aTAM, tiles attach only in locations with at least a certain number of correct bonds (here, two) and never detach. In the kTAM, tiles attach with rates dependent only on concentration and detach with rates dependent on the number of correct bonds. A few examples of attachment and detachment rates for given crystals are shown, omitting the common factor $\hat{k}_{\mathrm{f}}$. The right side shows the same crystals at a later time; in the aTAM, this is the unique and final result of tile attachments, whereas in the kTAM it is one possible state that can continue to grow or shrink. In the kTAM, a crystal can also contain mismatched tiles; a mismatched tile is highlighted by a black circle in the bottom right crystal.
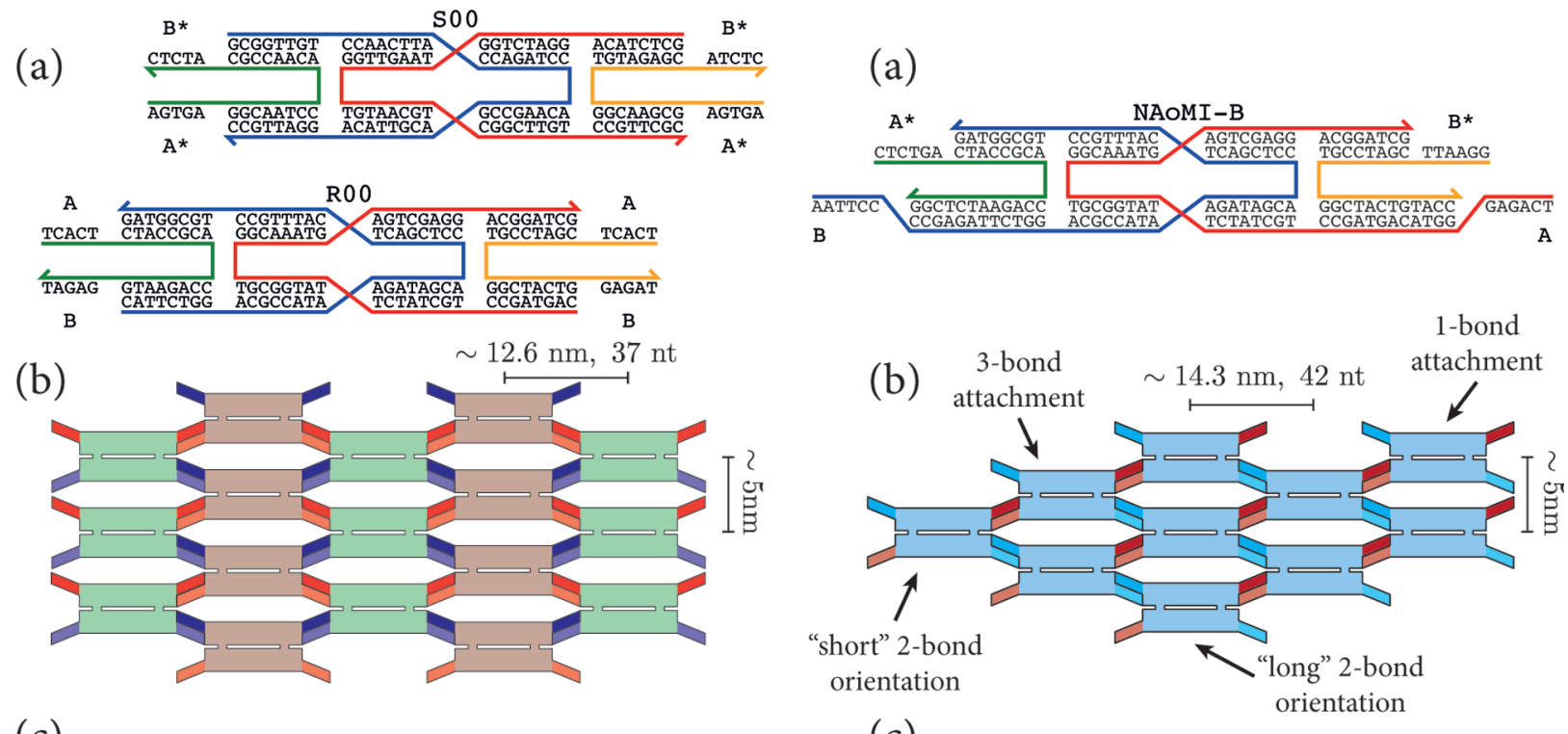

(c)
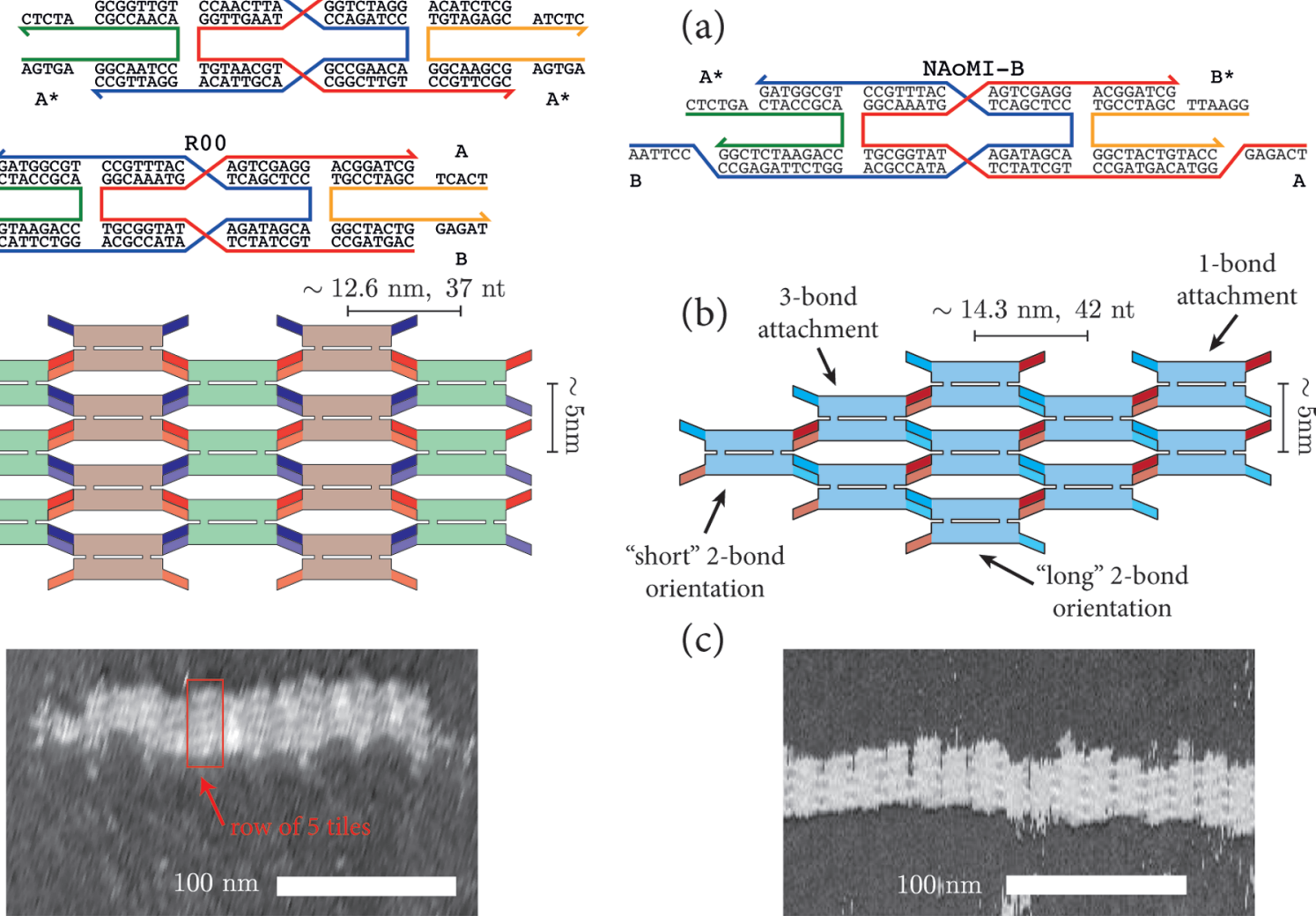

(c)

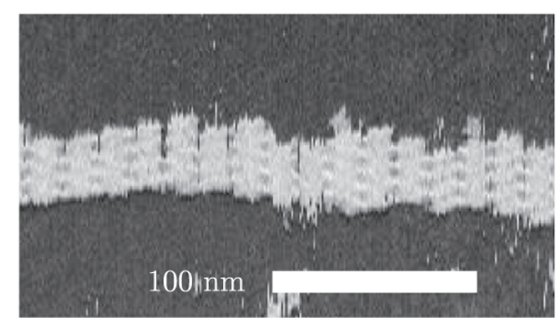

Figure 2. DNA tile structures and their resultant lattices for R00/S00 (left side of figure) and NAoMI-B (right side) systems. (a) Shows sequence designs of tiles: $\mathrm{A}, \mathrm{A} * \mathrm{~B}$, and $\mathrm{B}^{*}$ represent symbolic sticky ends, with * denoting complements and barbs indicating $3^{\prime}$ ends. (b) Shows example lattice structures resulting from perfect (no-mismatch) growth, along with examples of the "short" and "long" orientations of tiles attached by two bonds. (c) Shows example AFM images of lattices. 


\section{EXPERIMENTAL METHODS}

2.1. System Description. The systems described by the kinetic model are typically implemented with DNA tile structures similar to those shown in Figure 2. For example, in these experiments, we used two previously published tile systems that had two different molecular structures. The first was R00/S00, a system of two tiles that form a two-dimensional (2D) lattice. ${ }^{11}$ The second was NAoMI-B, ${ }^{12}$ a system with a single tile type that assembles in solution into three-dimensional (3D) tubes, but in our experiment formed into 2D lattices owing to association with the mica surface. ${ }^{13}$ The R00/S00 tiles have a DAO-E molecular structure: two crossovers between helices (Double), Antiparallel strand orientations (a strand in one helix has the opposite orientation after crossing over to the other helix), an Odd number of half-turns between crossover points in a single tile, and an Even number of half-turns between crossovers in adjacent tiles. NAoMI-B has a DAO-O structure, which is similar except with an odd number of half-turns between crossovers in adjacent tiles (see Figure $2 \mathrm{a}$ ). ${ }^{4,13,14}$ Both of these systems have similar properties, using tiles with four binding sites comprising five-base (R00/SO0) or six-base (NAoMI-B) single-stranded sticky ends that attach to complementary sticky ends on other tiles.

The kinetic model has been widely used as a quantitative model to investigate tile systems, ${ }^{15-17}$ and with some extensions (sequencedependent $\Delta G_{\mathrm{se}}^{\circ}$ s, varying concentrations), has been used in numerous computational simulations of crystal growth. ${ }^{11,18-20}$ Algorithmic growth behavior suggested by the KTAM and design techniques like proofreading ${ }^{15}$ that have been based on the model have translated well into experimental findings, ${ }^{6,719}$ but no investigation has been done directly into the model's mechanistic assumptions.

In particular, the kinetic model makes the following simplifying assumptions or idealizations: ${ }^{10}$

1 Free monomer concentrations remain constant during growth.

2 Crystals are perfectly rectilinear, $2 \mathrm{D}$, and free of defects, with single tiles that attach and detach one at a time.

3 Attachment rates are constant and equal regardless of the number of correct or incorrect bonds for a tile at the attachment site, as shown in eq 2.

4 Detachment rates are exponentially dependent on (a) the number of correct attachments the tile has to the crystal, as shown in eq 2 , and (b) on nothing else.
Assumption 1, while not true for growth in general, can be approximated by using seeded nucleation with a small enough concentration of seeds in comparison to tiles and a short enough period of sufficiently slow growth. ${ }^{6,17,20}$ Assumption 2 is beyond the scope of this paper; it is worth mentioning that we were able to obtain data without interference from lattice defects or multiple attachments, though violations of the assumptions have been seen., ${ }^{5,711}$ Assumptions 3 and 4 are of primary interest to us here, as there are a number of physical effects that could cause experiments to deviate from the assumptions. It is possible, for example, that electrostatic or steric effects in the molecular structure of the lattice could impact attachment and detachment rates in different ways, depending upon the location of the binding sites in the lattice or other factors. Another specific example is that tiles attaching by two bonds in both of our tile systems can have two different orientations of attachment to the lattice (Figure $2 \mathrm{~b}$ ); entropic or electrostatic effects could cause these orientations to have different rates. Any of these effects could violate 3 and $4 \mathrm{~b}$. Tiles with multiple bonds to the lattice could also attach and detach via processes that might violate the simple additive assumption of $4 \mathrm{a}$, such as detachment and attachment of a single bond in a tile with four bonds.

2.2. Atomic Force Microscopy. In our experiments, we examined assumptions 3 and 4 by using fluid tapping-mode atomic force microscopy (AFM) to create movies of individual tiles attaching and detaching in lattices. The lattices were in a fluid environment with free monomers but were weakly bound to mica for imaging while growing; ${ }^{21}$ while most systems have previously been grown with lattices in solution, there have been systems successfully grown on surfaces in a similar fashion. ${ }^{22-24}$ With a system in growth-biased conditions, we were able to create movies of individual crystals growing rapidly on the surface (Figure 3a and movies 3 and 4 in Supporting Information [SI]); however, attachment of individual tiles took place too quickly for us to distinguish single-tile changes, and in any case, there would have been too few detachments to obtain sufficient statistics. By instead imaging near an equilibrium that we reached via adjustment of temperature, tile concentration, and other parameters (discussed later), we were able to observe distinct, single-tile attachments and detachments on single crystals, and compile statistics of these changes as a function of the number of bonds and tile orientation.

Throughout the experiment, we used TAE $/ \mathrm{Mg}^{2+}$ buffer $(40 \mathrm{mM}$ Tris, $20 \mathrm{mM}$ acetic acid, $1 \mathrm{mM}$ EDTA, $12.5 \mathrm{mM} \mathrm{Mg}$ acetate) and PAGE-purified strands (Integrated DNA Technologies). For R00/S00,

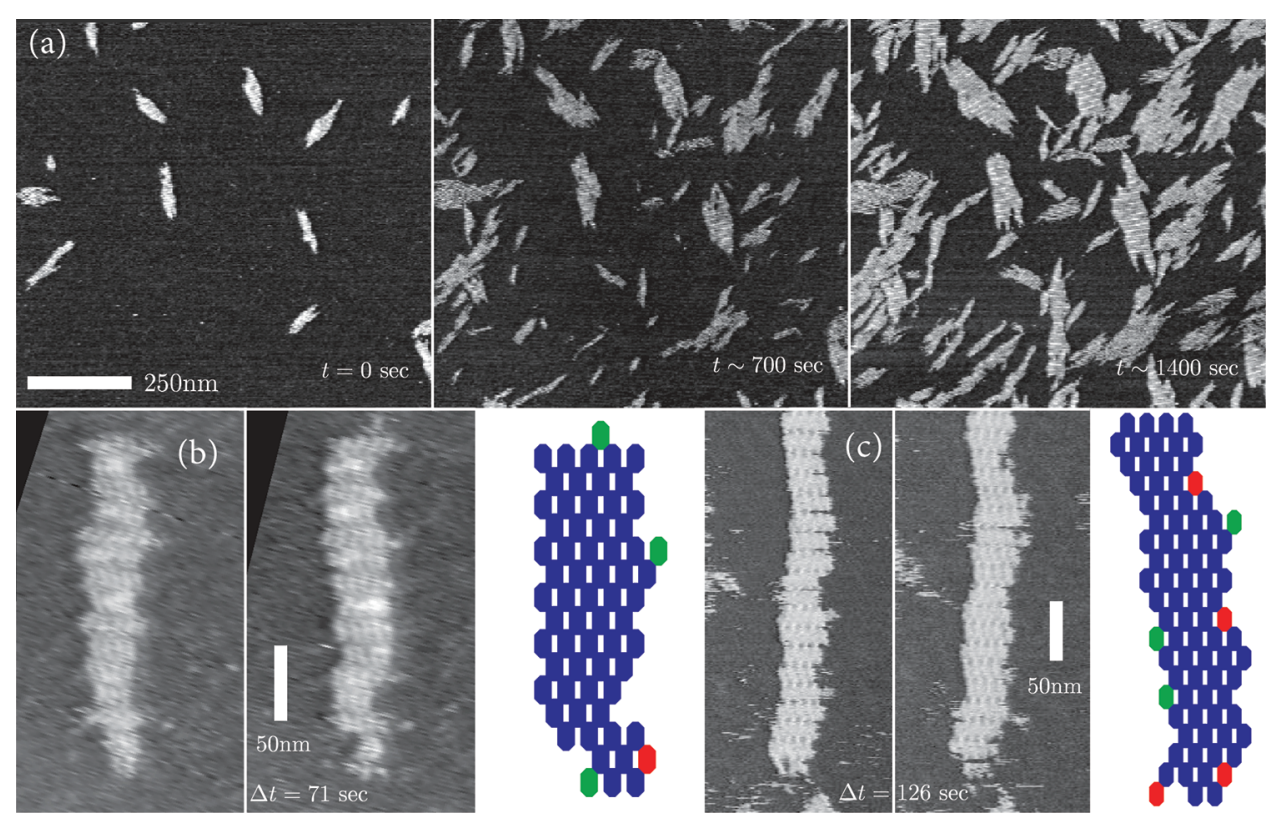

Figure 3. AFM images of R00/S00 and NAoMI-B systems: (a) shows several frames from a movie of quickly growing R00/S00 crystals. (b) with R00/S00 and (c) with NAoMI-B show adjacent frames in a near equilibrium movie (movies 1 and 2 in SI) and their interpretation: blue tiles are present in both frames, while green tiles attached and red tiles detached from one frame to the next. 
we annealed mixes of strands ( $25 \mathrm{nM}$ each) in buffer for each of the two individual tiles separately with a temperature decrease from 90 to $20{ }^{\circ} \mathrm{C}$ at $1{ }^{\circ} \mathrm{C} / \mathrm{min}$, starting with $90{ }^{\circ} \mathrm{C}$ incubation for $5 \mathrm{~min}$; crystals could only grow when these were later mixed. For NAoMI-B, all strands were mixed together at $1 \mu \mathrm{M}$ per strand and annealed with a 70 min linear temperature decrease from 90 to $20{ }^{\circ} \mathrm{C}$; this created nanotubes that were then diluted to a $50 \mathrm{nM}$ per tile concentration. As in previous work, ${ }^{13}$ nearly all DNA tile nanotubes break open and unfold during imaging, so that they appear as thin strips of $2 \mathrm{D}$ tile crystals. Imaging was performed on a MultiMode AFM (Bruker) with a Nanoscope IIIa controller, with temperature held at $33.4{ }^{\circ} \mathrm{C}$ by an in-stage heater (R00/S00) or at $32{ }^{\circ} \mathrm{C}$ (NAoMI-B) by a space heater heating the entire room. Around $40 \mu \mathrm{L}$ buffer was deposited on freshly cleaved mica; this was allowed to equilibrate in temperature in the AFM and was then imaged without any tiles. Around $5 \mu \mathrm{L}$ of each annealed tile mix (two for R00/S00, one for NAoMI-B) was usually added to this buffer while still imaging. While surface binding effects, evaporation, and our AFM's open fluid cell design made it difficult to know the effective tile concentrations in the sample chamber during AFM imaging, relative concentrations were adjusted such that the crystals were near equilibrium, thus simplifying analysis, and were kept equal across different tile types for R00/S00. Imaging speed was kept as fast as possible while still allowing individual tile resolution, which for our AFM resulted in a speed of around $80 \mathrm{~s} /$ frame at a scale of around 600 $\mathrm{nm}$ with a resolution of 512 pixels per scan line and 512 scan lines per image. Images of five crystals from three movies were used: one long, large movie with two crystals for R00/S00, and two shorter movies, one with two crystals and one with a single crystal, for NAoMI-B.

Our ability to observe individual tile attachments and detachments as they occurred was made possible by a number of coincidental advantages found by our AFM use in prior experiments with DNA tiles. In fluid-tapping mode, free tiles were able to remain in our buffer solution rather than binding to mica, allowing us to achieve a suitable concentration of free tiles in solution for growth. The ability to reliably image in the 30$40{ }^{\circ} \mathrm{C}$ range allowed us to maintain a temperature near equilibrium. Adjusting buffer salt concentrations would have allowed us to ensure that reasonably sized lattices would bind to mica while monomers remained in solution, thus taking advantage of salt modulation of mica-DNA binding; ${ }^{21,25}$ in practice, we found that our buffer's salt concentrations were already acceptable. By combining control of temperature and tile concentrations, we were able to tune attachment and detachment rates such that individual events could be resolved at our imaging speed. We additionally used nanoAnalytics analog Q-control (Asylum Research) and tuning of AFM parameters in order to minimize any damage to the lattices during imaging.

\section{ANALYSIS}

To analyze the resulting movies, as AFM images tend to vary considerably in contrast, distortion, and sample location from frame to frame, we manually translated visible crystals into lattice structures by counting the number of tiles in each visible "row" of tiles, and recording the offset of this row from adjoining rows. Lattices from subsequent frames were then automatically aligned, and combined with estimated in-frame position data taken from a combination of average lattice spacing and crystal position and angles. The position and timing of events at each lattice position were derived from these data. Occasionally, tiles adjacent to each other would both attach or both detach between the same frames, creating ambiguous events, but this did not affect the results significantly and was compensated for by assuming that the most probable order of events was what actually took place: that is, the detaching tiles that were attached by the least number of bonds in the older frame detached first, and the attaching tiles that could attach by the most number of bonds attached first.

From these data, consisting of 115 identified attachment and detachment events for R00/S00 and 96 for NAoMI-B (not including tiles that stayed attached or lattice sites that remained empty), our challenge is to separately infer the on-rate and off-rate for each type of crystal site, and then together (globally) to extract $G_{\mathrm{mo}} G_{\mathrm{se}}$ and $\hat{k}_{\mathrm{f}}$. The agreement (or lack of it) between the separate and global fits will allow us to assess assumptions 3 and 4 .

Each position in a lattice can be in two states: filled (F) or empty (E). From one frame to the next, we can therefore have a filled position remained filled (FF), an empty position remain empty (EE), a filled position become empty (FE), or an empty position become filled (EF). Each pair of measurements of each position in the lattice from one frame to the next constitutes a Bernoulli trial $i$ with a probability $p_{i}$ of remaining the same (FF, EE) and a probability $1-p_{i}$ of changing (FE, EF). Depending upon the initial state, these probabilities are dependent upon the time between the two measurements $\Delta t_{i}$ (which can take into account dropped frames, position within the frame, and AFM irregularities, rather than just frame rate), and an attachment or detachment rate $\lambda_{\theta}^{c}$, where $c$ is either EF or FE (determined by the initial state of the position) and $\theta$ denotes the presumed rateaffecting parameters of the set of locations we're considering: the number of bonds surrounding the position, and when accounting for it, the orientation of two-bond positions. As we assume these rates are constant for given parameters, the probability of a trial $i$ over a time $\Delta t_{i}$ resulting in no change is $p_{i}=\mathrm{e}^{-\lambda_{\theta_{i}}^{c_{i}} \Delta t_{i}}$.

For a set of trials with the same $\theta$ and starting state (empty or full), we can use Bayesian inference to provide the probability of a rate $\lambda_{\theta}^{c}$ given our data. The a posteriori probability of our data- a set of trials $N C_{\theta, c}$ where a location remained unchanged ( $\mathrm{FF}$ or $\mathrm{EE}$ ), and a set of trials $C_{\theta, c}$ where a location changed (FE or EF) - with an assumed rate $\lambda_{\theta}^{c}$ is simply the product of the individual Bernoulli distribution probabilities:

$$
p\left(\operatorname{datal} \mid \lambda_{\theta}^{c}\right)=\prod_{i \in N C_{\theta, c}} \mathrm{e}^{-\lambda_{\theta}^{c} \Delta t_{i}} \prod_{i \in C_{\theta, c}}\left(1-\mathrm{e}^{-\lambda_{\theta}^{c} \Delta t_{i}}\right)
$$

Bayesian inference would therefore assert that the probability of a rate $\lambda_{\theta}^{c}$ given our data is

$$
p\left(\lambda_{\theta}^{c} \text { ldata }\right)=\frac{p\left(\lambda_{\theta}^{c}\right)}{p(\text { data })}\left[\prod_{i \in N C_{\theta, c}} \mathrm{e}^{-\lambda_{\theta}^{c} \Delta t_{i}} \prod_{i \in C_{\theta, c}}\left(1-\mathrm{e}^{-\lambda_{\theta}^{c} \Delta t_{i}}\right)\right]
$$

Using the uniform prior $p\left(\lambda_{\theta}^{c}\right)$, both our prior and our likelihood $p$ (data) are independent of $\lambda_{\theta}^{c}$ and thus can be considered a constant normalization factor to our probability. Thus, our inferred rate of attachment or detachment given our set of data is the $\lambda_{\theta}^{c}$ that maximizes $p\left(\right.$ datal $\left.\lambda_{\theta}^{c}\right)$ from eq 3 . Our probabilities are given by normalizing $p\left(\right.$ datal $\left.\lambda_{\theta}^{c}\right)$ over a reasonable range of $\lambda_{\theta}^{c}$ values (such that the a posteriori distribution drops to approximately zero on the boundaries) and then using it as a probability density function.

This method provides us with the rates of attachment and detachment per lattice position for a given set of parameters. kTAM's assumptions 3 and 4 provide that these rates should be related by eq 2 ; in other words, for some $\hat{k}_{\mathrm{f}}, G_{\mathrm{mc}}$ and $G_{\mathrm{se}}$, we should have

$$
\begin{aligned}
& \lambda_{\theta}^{\mathrm{EF}}=\hat{k}_{\mathrm{f}} \mathrm{e}^{-G_{\mathrm{mc}}} \\
& \text { and } \\
& \lambda_{\theta}^{\mathrm{FE}}=\hat{k}_{\mathrm{f}} \mathrm{e}^{-b(\theta) G_{\text {se }}}
\end{aligned}
$$

Using Bayesian inference again, we can obtain $G_{\mathrm{se}}, G_{\mathrm{mc}}$ and $\hat{k}_{\mathrm{f}}$ for a given data set of detachments with varying numbers of 
bonds by using $G_{\mathrm{se}}, G_{\mathrm{mo}}$ and $\hat{k}_{\mathrm{f}}$ as parameters, using eq 5 to derive detachment rates for different $\theta$ parameters, and including all data in the sets $N C$ and $C$ :

$$
\begin{aligned}
& p\left(G_{\mathrm{se}}, G_{\mathrm{mc}}, \hat{k}_{\mathrm{f}} \text { ldata }\right) \\
& =\frac{p\left(G_{\mathrm{se}}, G_{\mathrm{mc}}, \hat{k}_{\mathrm{f}}\right)}{p(\text { data })}\left[\prod_{i \in N C} \exp \left[-\Delta t_{i} \lambda_{\theta_{i}}^{c_{i}}\left(G_{\mathrm{se}}, G_{\mathrm{mc}}, \hat{k}_{\mathrm{f}}\right)\right]\right. \\
& \left.\quad \times \prod_{i \in C}\left(1-\exp \left[-\Delta t_{i} \lambda_{\theta_{i}}^{c_{i}}\left(G_{\mathrm{se}}, G_{\mathrm{mc}}, \hat{k}_{\mathrm{f}}\right)\right]\right)\right]
\end{aligned}
$$

As we can again use a uniform prior, the probability density for $G_{\mathrm{se}}, G_{\mathrm{mc}}$ and $\hat{k}_{\mathrm{f}}$ can be derived by ignoring $p\left(\Delta G_{\mathrm{se}}^{\circ}, G_{\mathrm{mo}} \hat{k}_{\mathrm{f}}\right) /$ $p$ (data) and normalizing $p\left(G_{\mathrm{se}}, G_{\mathrm{mc}}, \hat{k}_{\mathrm{f}}\right.$ Idata) over a sufficiently large parameter space.

In order to verify that our analysis produced accurate results when presented with data fitting the kinetic model, the entire analysis, with the exception of the manual data entry, was applied to simulated data taken from the Xgrow kTAM simulator. ${ }^{26}$ Parameters were chosen to match conditions in our experimental results, the amount of data was chosen to be similar, and the output was modified to mimic the line-scanning of an AFM. The results (Figure $4 \mathrm{~b}, \mathrm{~d}$ ) suggested our analysis methods were sound in the face of finite scanning rates and multiple events.

\section{RESULTS AND DISCUSSION}

Our results, shown in Figure 4, were compiled separately for R00/S00 and NAoMI-B. Ignoring the possibility of incorrect bonds and lattice defects in the system (assumption 2), and assuming constant monomer concentrations (assumption 1), our results largely suggest that assumptions 3 (equal attachment rates) and $4 \mathrm{a}$ (detachment rates exponentially dependent on the number of bonds) of the kTAM are valid within our experimental error. Additionally, while observing relatively large error bounds owing to the smaller amount of data for each orientation, we found that tile orientation did not alter detachment rate beyond experimental error, and thus did not violate assumption $4 \mathrm{~b}$ (detachment rates dependent upon nothing but number of bonds).

As for the consistency of the quantitative results, it is worth remembering that NAoMI-B uses 6 nt sticky ends, whereas R00/S00 uses 5 nt sticky ends. Using the rough approximation that $G_{\text {se }}$ scales linearly with the number of nucleotides, this suggests that the NAoMI-B $G_{\text {se }}$ should be around 1.2 times that of the R00/S00 $G_{\mathrm{se}}$. Our results give a ratio of around $1.14 \pm$ 0.15 , in line with this approximated expectation.

There were several key differences between our experimentally observed data and simulated data, many of which likely originate from differences in the relative numbers of events observed and ultimately in overall crystal "shape." In particular, data from simulation, while resulting in similar detachment rates, had significantly more empty lattice locations with three or four connecting bonds $(b=3$ or $b=4)$. These sites are the result of a crystal that is, subjectively, more "spindly" and less compact than those we found experimentally, with long "arms" of tiles. Whether "spindly" crystals actually form is a question that would require a different experiment; however, regardless of their formation, it is quite likely that crystals of that shape would not cleanly bind to mica, or would produce AFM images unsuitable for analysis; our experiment, therefore, inadvertently selected against crystals of this form and instead selected for relatively solid, compact crystals that were easily imaged. In a related shape issue, the fact that imaged NAoMI-B crystals originated from nanotubes in solution meant that all observed crystals were long, narrow ribbons, resulting in no data for short-orientation two-bond attachments, as our data never included the ends of the crystals.

Our analysis of attachment and detachment rates involved only statistics of detachment and attachment for single tiles. The rates derived from such an analysis would ideally be independent of crystal shape, with only the size of the error bounds differing, owing to differences in the number of events. In some cases, however, the significantly smaller number of events likely resulted in anomalous observations having a large effect on attachment and detachment rates. For R00/S00, the attachment rate for $b=4$ is calculated from only 5 events ( 2 empty-to-empty and 3 empty-to-full), and is significantly higher than expected by kTAM. For NAoMI-B, the attachment rate for $b=3$ is similarly calculated by only 10 events, and is also significantly higher than expected. By comparison, $b=3$ for R00/S00 involved 54 events, and was in line with expectations.

With such events made rare by crystal shape, the chance of the rates being affected by errors in image interpretation, anomalous imaging results, or erroneous assumptions about the order of tile attachment is significantly increased. We expect that this accounts for the discrepancies seen in attachment rates, as all unexpected attachment rates have very few recorded events.

In addition to the directly obtainable results, we can also give rough estimates of other parameters. Bayesian inference suggested $\hat{k}_{\mathrm{f}}$ values of $0.024 / \mathrm{M} / \mathrm{s}$ and $0.053 / \mathrm{M} / \mathrm{s}$ for $\mathrm{R} 00 / \mathrm{S} 00$ and NAoMI-B, respectively. If we assume what should be a reasonable order-of-magnitude estimation of a $10 \mathrm{nM}$ tile concentration, this results via eqs 1 and 2 in an $\alpha \approx-15$ and $k_{\mathrm{f}} \approx 10^{6} / \mathrm{M} / \mathrm{s}$. Such a $k_{\mathrm{f}}$ value is on the same order of the $6 \times$ $10^{5} / \mathrm{M} / \mathrm{s}$ used in ref 10 , but the $\alpha$ is very different than the $+\ln (20)$ used there for simulations of growth in solution. ${ }^{10}$ $R T \alpha$ can be interpreted as a free energy adjustment that remains constant for all attached tiles, as opposed to the $\Delta G_{\text {se }}^{\circ}$ which is scaled with the number of bonds. While the positive $\alpha$ for growth in solution can be interpreted as due to the loss of rotational entropy when a tile binds to the crystal, negative $\alpha$ corresponds to an energetically favorable contribution to attachment in all cases, as could be expected for growth on mica where all tiles involved bind to the mica surface. ${ }^{21}$

In comparing our results to other experiments, it is necessary to convert standard free energies to our unitless $G_{\mathrm{se}}=\Delta G_{\mathrm{se}}^{\circ} / R T$ (eqs 1 and 2), and in many cases make a change in sign per our convention. The $G_{\text {se }}$ we calculate from the statistics is significantly lower than that found by experiments in solution rather than on a mica surface; this corresponds to a significantly "weaker" binding energy contribution for individual sticky ends. In particular, Schulman et $\mathrm{al}^{20}$ measured an insolution $\Delta G_{2 \mathrm{se}}^{\circ}=-9.43 \pm 0.21 \mathrm{kcal} / \mathrm{mol}$ at $37{ }^{\circ} \mathrm{C}$ for two bonds, corresponding to $G_{\mathrm{se}}=7.7 \pm 0.17$. Nangreave et al, ${ }^{27}$ using a different form of tile with 6 nt sticky ends, found a $\Delta G_{\text {se }}^{\circ}$ on the order of -3 to $-6 \mathrm{kcal} / \mathrm{mol}$, depending upon the form of attachment, resulting in a $G_{\text {se }}$ of between 6 and 10 . By comparison, our rates of $G_{\mathrm{se}}=1.55$ and $G_{\mathrm{se}}=1.77$ are extremely low. Indeed, a simple order of magnitude comparison of kTAM-expected detachment rates derived from the 7.7 value of Schulman would suggest that, were the $\Delta G_{\text {se }}^{\circ}$ in our on-mica conditions even close to so large, our entire experiment, and viewing growth with any level of detail at all, would be infeasible: the difference in rates between even tiles attached by two and three bonds would be on the order of $\mathrm{e}^{-2 \cdot 7.7} / \mathrm{e}^{-3 \cdot 7.7} \approx 2200$. 
(a)

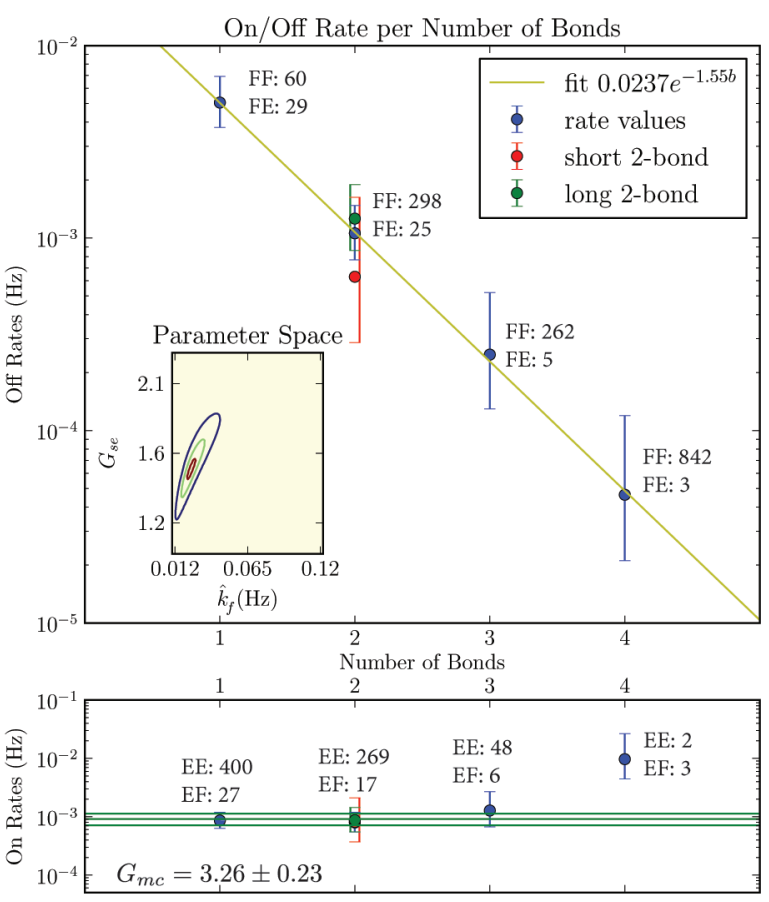

(b)

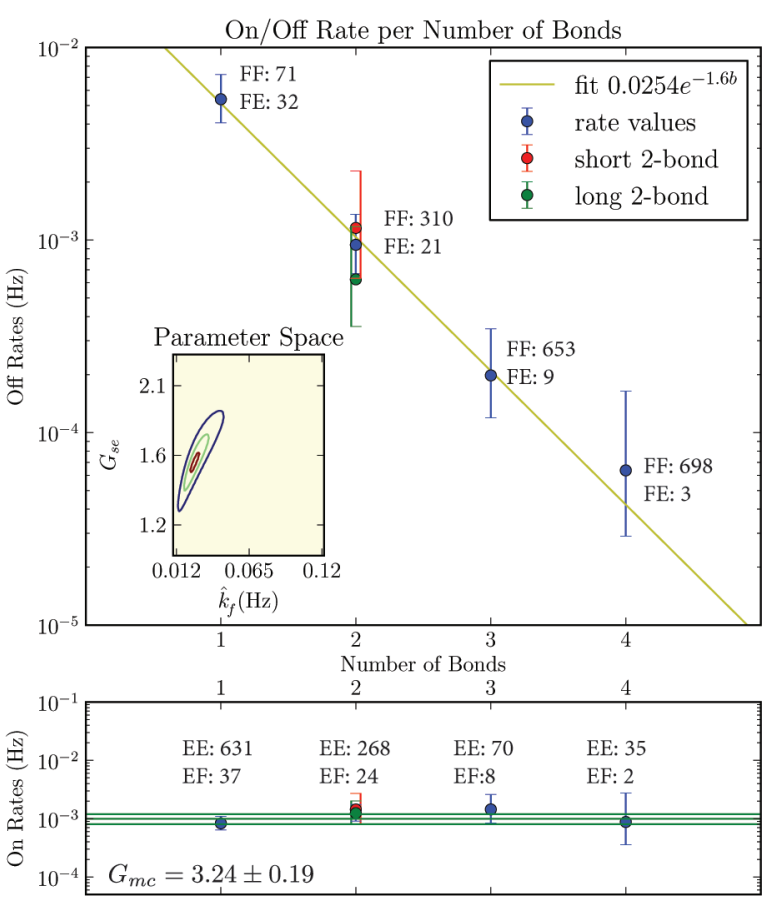

(c) NAoMI-B Experimental Results
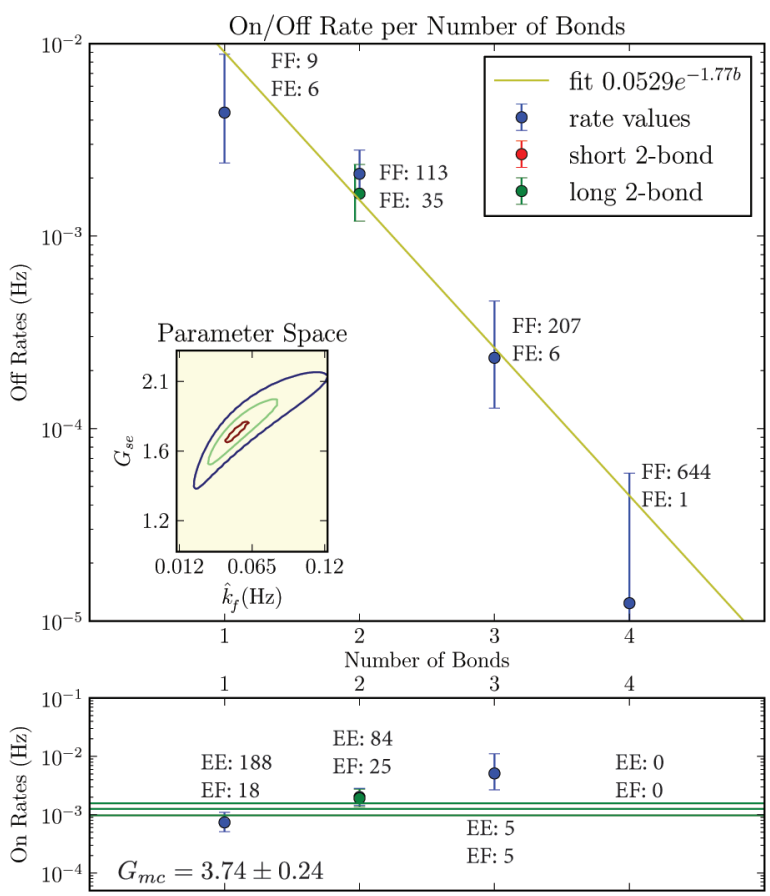

(d)

NAoMI-B Simulation Results
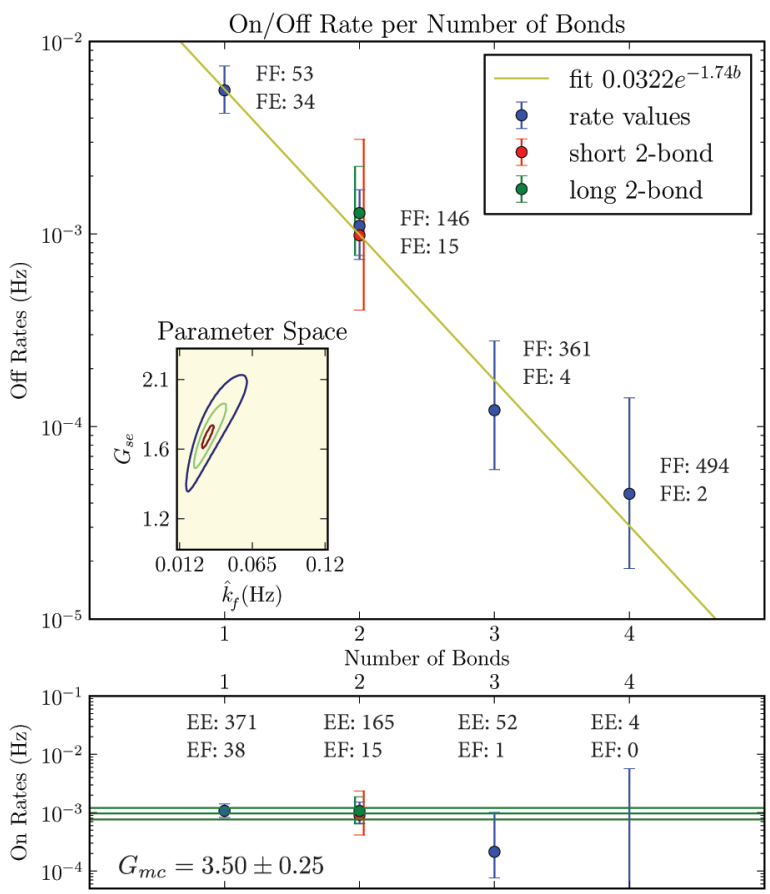

Figure 4. Plots of detachment and attachment rates for R00/S00 (left top) and NAoMI-B (right top) lattices. Bottom figures show simulation data with similar parameters. Insets in detachment rate plots show activation rate $/ G_{\text {se }}$ parameter space, with lines representing $p=0.1,0.5$, and 0.9 . Lines in attachment rate plots show mean and $90 \%$ confidence interval for $G_{\mathrm{mc}}$.

There are a number of possible factors involving DNA binding with mica and DNA hybridization near a mica surface which could explain this discrepancy. A difference in binding for ssDNA vs dsDNA to mica, for example, could result in a modified $G_{\text {se }}$ for sticky ends, as two free, singlestranded sticky ends could have a stronger binding to mica than the bound, double-stranded combination of the two ends. Also, while DNA hybridization near a mica surface has not been widely studied, it is reasonable to expect that there could be significant differences from hybridization in solution: at the very least, the "salt bridge" of divalent cations (in our case, $\mathrm{Mg}^{2+}$ ) between the DNA and mica would result in a very different and likely nonhomogenous ionic environment. ${ }^{21}$ 


\section{CONCLUSIONS}

With this work, we have shown that we can make single-tileresolution AFM movies of single DNA tile system crystals at a sufficient frame rate to provide a consistent view of the steps involved in assembly. While we have used these movies to verify some key assumptions of the kTAM, at least for growth on a surface, the technique raises the prospect of examining growth on a surface in general at a level that has not previously been possible. For example, AFM movies could be used to examine the growth of algorithmic tile systems, possibly allowing insight into the mechanisms of both errors and error-reducing methods such as proofreading ${ }^{15}$ and snaked proofreading. ${ }^{16}$

It is worth noting that if the $G_{\text {se }}$ for tile detachment on a mica surface is indeed significantly lower than that in solution, as we have found, it may significantly increase error rates for algorithmic tile systems grown on mica surfaces, with the kinetic trapping model ${ }^{10}$ suggesting a possible error rate of upward of $30 \%$ for $G_{\mathrm{se}}=1.6$ and Xgrow simulations suggesting an error rate for the binary counter system from Barish et al. ${ }^{6}$ too high to be measured. However, for the examination of the mechanisms behind errors this could end up being a benefit, with the higher error rate providing significantly more data on what might otherwise be errors in a relatively accurate system. As algorithmic systems can often be reliant on perfect, errorfree assembly, the ability to examine the systems, especially those that involve proofreading schemes, in an environment that significantly increases error rate could be vital in understanding how to further reduce errors.

\section{ASSOCIATED CONTENT}

\section{S Supporting Information}

Several AFM movies of nonequilibrium and equilibrium crystal growth:

- Movie 1: equilibrium conditions with R00/S00; $25 \mathrm{nM}$ each of R00/S00 were annealed separately, mixed in the AFM sample stage buffer during imaging, and imaged at $33.4{ }^{\circ} \mathrm{C}$; the movie has a $\sim 560 \mathrm{~nm} \times 525 \mathrm{~nm}$ field of view for $1088 \mathrm{~s}$ at a frame rate of $\sim 73 \mathrm{~s} /$ frame.

- Movie 2: equilibrium conditions with NAoMI-B; $50 \mathrm{nM}$ of NAoMI-B was annealed and imaged at $32{ }^{\circ} \mathrm{C}$; the movie has a $\sim 430 \mathrm{~nm} \times 425 \mathrm{~nm}$ field of view for $1012 \mathrm{~s}$ at a frame rate of $\sim 126 \mathrm{~s} /$ frame.

- Movie 3: growth-biased conditions for R00/S00; $100 \mathrm{nM}$ each of R00 and S00-2J (S00 with added hairpins for labeling purposes creating a striped lattice, also known as $\hat{B}$ in Figure $4 a-c$ and Supporting Information of ref 4.) were annealed separately. Five microliters of each was mixed in $30 \mu \mathrm{L}$ of the AFM sample stage buffer during imaging, and was imaged at $32{ }^{\circ} \mathrm{C}$. The movie has a $\sim 2 \mu \mathrm{m}$ field of view at a frame rate of $\sim 70 \mathrm{~s} /$ frame.

- Movie 4: growth-biased conditions for NAoMI-B; $200 \mathrm{nM}$ of NAoMI-B was annealed from 90 to $40{ }^{\circ} \mathrm{C}$ at $1{ }^{\circ} \mathrm{C} / \mathrm{min}$, and was then added directly to unheated buffer in the AFM and imaged at room temperature. The movie has a $\sim 4 \mu \mathrm{m}$ field of view at $\sim 84 \mathrm{~s} /$ frame. The concentration of tiles was doubled during the two frames that exhibit extensive line artifacts.

For presentation purposes, equilibrium movie image contrast levels were manually adjusted, with black and white being set at the bottom of histogram peaks for surface and tile heights, respectively, and gray being set midway between the two peaks. Images were then manually shifted (but not stretched) to adjust for AFM drift. Growth-biased movies underwent the same process with the addition of manual stretching of images to adjust for AFM distortion. This material is available free of charge via the Internet at http://pubs.acs.org.

\section{AUTHOR INFORMATION}

\section{Corresponding Author}

winfree@caltech.edu

\section{Present Address}

${ }^{\perp}$ Cell and Developmental Biology, University of Michigan, Ann Arbor, MI 48109-2200, United States

\section{Notes}

The authors declare no competing financial interest.

\section{ACKNOWLEDGMENTS}

We thank Christina Wright for prototype software for analyzing AFM movies, and Shaun Lee for discussions regarding AFM movies of DNA tiles. We acknowledge National Science Foundation (NSF) Awards 0832824 ("The Molecular Programming Project") and 0622254.

\section{REFERENCES}

(1) Vauthey, S.; Santoso, S.; Gong, H.; Watson, N.; Zhang, S. Proc. Natl. Acad. Sci. U.S.A. 2002, 99, 5355-5360.

(2) Nam, K. T.; Kim, D.-W.; Yoo, P. J.; Chiang, C.-Y.; Meethong, N.; Hammond, P. T.; Chiang, Y.-M.; Belcher, A. M. Science 2006, 312, $885-888$.

(3) Nasalean, L.; Baudrey, S.; Leontis, N. B.; Jaeger, L. Nucleic Acids Res. 2006, 34, 1381-1392.

(4) Winfree, E.; Liu, F.; Wenzler, L. A.; Seeman, N. C. Nature 1998, 394, 539-544.

(5) Barish, R. D.; Rothemund, P. W. K.; Winfree, E. Nano Lett. 2005, $5,2586-2592$.

(6) Barish, R. D.; Schulman, R.; Rothemund, P. W. K.; Winfree, E. Proc. Natl. Acad. Sci. U.S.A. 2009, 106, 6054-6059.

(7) Fujibayashi, K.; Hariadi, R.; Park, S. H.; Winfree, E.; Murata, S. Nano Lett. 2008, 8, 1791-1797.

(8) Chworos, A.; Severcan, I.; Koyfman, A. Y.; Weinkam, P.; Oroudjev, E.; Hansma, H. G.; Jaeger, L. Science 2004, 306, 2068-2072.

(9) Wang, H. An Unsolvable Problem on Dominoes; Harvard Computation Laboratory: Cambridge, MA, 1962.

(10) Winfree, E. Simulations of Computing by Self-Assembly; California Institute of Technology: Pasadena, CA, 1998.

(11) Rothemund, P. W. K.; Papadakis, N.; Winfree, E. PLoS Biol. 2004, 2, e424.

(12) Hariadi, R. F. Non-equilibrium Dynamics of DNA Nanotubes.

Ph.D. Thesis; California Institute of Technology: Pasadena, CA, 2011.

(13) Rothemund, P. W. K.; Ekani-Nkodo, A.; Papadakis, N.; Kumar, A.; Fygenson, D.; Winfree, E. J. Am. Chem. Soc. 2004, 126, 1634416352.

(14) Fu, T. J.; Seeman, N. C. Biochemistry 1993, 32, 3211-3220, PMID: 8461289.

(15) Winfree, E.; Bekbolatov, R. In DNA Computing; Chen, J., Reif, J., Eds.; Lecture Notes in Computer Science; Springer: Berlin/ Heidelberg, 2004; Vol. 2943; pp 1980-1981.

(16) Chen, H.-L.; Goel, A. In DNA Computing; Ferretti, C., Mauri, G., Zandron, C., Eds.; Lecture Notes in Computer Science; Springer: Berlin/Heidelberg, 2005; Vol. 3384; pp 702-707.

(17) Schulman, R.; Winfree, E. In DNA Computing; Ferretti, C., Mauri, G., Zandron, C., Eds.; Lecture Notes in Computer Science; Springer: Berlin/Heidelberg, 2005; Vol. 3384; pp 672.

(18) Fujibayashi, K.; Murata, S. IEEE Trans. Nanotechnol. 2009, 8, 361-368.

(19) Chen, H.-L.; Schulman, R.; Goel, A.; Winfree, E. Nano Lett. 2007, 7, 2913-2919. 
(20) Schulman, R.; Winfree, E. Proc. Natl. Acad. Sci. U.S.A. 2007, 104, 15236-15241.

(21) Pastré, D.; Piétrement, O.; Fusil, S.; Landousy, F.; Jeusset, J.; David, M.-O.; Hamon, L.; Le Cam, E.; Zozime, A. Biophys. J. 2003, 85, $2507-2518$

(22) Hamada, S.; Murata, S. Angew. Chem. 2009, 121, 6952-6955.

(23) Sun, X.; Hyeon Ko, S.; Zhang, C.; Ribbe, A. E.; Mao, C. J. Am. Chem. Soc. 2009, 131, 13248-13249, PMID: 19715316.

(24) Kim, B.; Amin, R.; Lee, J.; Yun, K.; Park, S. H. Chem. Commun. 2011, 47, 11053-11055.

(25) Bezanilla, M.; Manne, S.; Laney, D. E.; Lyubchenko, Y. L.; Hansma, H. G. Langmuir 1995, 11, 655-659.

(26) The Xgrow Simulator. http://www.dna.caltech.edu/Xgrow/.

(27) Nangreave, J.; Yan, H.; Liu, Y. Biophys. J. 2009, 97, 563-571. 